\title{
Orienting and separating distance-transitive graphs
}

\author{
Ítalo J. Dejter \\ University of Puerto Rico, Rio Piedras, PR 00936-8377, Puerto Rico
}

Received 3 October 2011, accepted 7 March 2012, published online 6 September 2012

\begin{abstract}
It is shown that exactly 7 distance-transitive cubic graphs among the existing 12 possess a particular ultrahomogeneous property with respect to oriented cycles realizing the girth that allows the construction of a related Cayley digraph with similar ultrahomogeneous properties in which those oriented cycles appear minimally "pulled apart", or "separated" and whose description is truly beautiful and insightful. This work is proposed as the initiation of a study of similar ultrahomogeneous properties for distance-transitive graphs in general with the aim of generalizing to constructions of similar related "separator" Cayley digraphs.
\end{abstract}

Keywords: Distance-transitive graph, ultrahomogeneous graph, Cayley graph.

Math. Subj. Class.: 05C62, 05B30, 05C20, 05C38

\section{Introduction}

A graph is said to be distance-transitive if its automorphism group acts transitively on ordered pairs of vertices at distance $i$, for each $i \geq 0[3,10,15]$. In this paper we deal mainly with finite cubic distance-transitive graphs. While these graphs are classified and very well-understood since there are only twelve examples, for this very restricted class of graphs we investigate a property called ultrahomogeneity that plays a very important role in logic, see for example [7, 18]. For ultrahomogeneous graphs (resp. digraphs), we refer the reader to $[5,9,11,17,19]$ (resp. [6, 8, 16]). Distance-transitive graphs and ultrahomogeneous graphs are very important and worthwhile to investigate. However, to start with, the following question is answered in the affirmative for 7 of the 12 existing cubic distance-transitive graphs $G$ and negatively for the remaining 5:

Question 1.1. If $k$ is the largest $\ell$ such that $G$ is $\ell$-arc-transitive, is it possible to orient all shortest cycles of $G$ so that each two oppositely oriented $(k-1)$-arcs of $G$ are just in two corresponding oriented shortest cycles?

E-mail address: ijdejter@uprrp.edu (Ítalo J. Dejter) 
The answer (below) to Question 1 leads to 7 connected digraphs $\mathcal{S}(G)$ in which all oriented shortest cycles of $G$ are minimally "pulled apart" or "separated". Specifically, it is shown that all cubic distance-transitive graphs are $\left\{C_{g}\right\}_{P_{k}}$-ultrahomogeneous, where $g=$ girth, but only the 7 cited $G$ are $\left\{\vec{C}_{g}\right\}_{\vec{P}_{k}}$-ultrahomogeneous digraphs and in each of these 7 digraphs $G$, the corresponding "separator" digraph $\mathcal{S}(G)$ is: (a) vertex-transitive digraph of indegree $=$ outdegree $=2$, underlying cubic graph and automorphism group as that of $G$; (b) $\left\{\overrightarrow{C_{g}}, \overrightarrow{C_{2}}\right\}$-ultrahomogeneous digraph, where $\overrightarrow{C_{g}}=$ induced oriented $g$-cycle, with each vertex taken as the intersection of exactly one such $\vec{C}_{g}$ and one $\vec{C}_{2} ;$ (c) a Cayley digraph. The structure and surface-embedding topology [2, 12, 20] of these $\mathcal{S}(G)$ are studied as well. We remark that the description of these $\mathcal{S}(G)$ is truly beautiful and insightful.

It remains to see how Question 1 can be generalized and treated for distance-transitive graphs of degree larger than 3 and what separator Cayley graphs could appear via such a generalization.

\section{Preliminaries}

We may consider a graph $G$ as a digraph by taking each edge $e$ of $G$ as a pair of oppositely oriented (or O-O) arcs $\vec{e}$ and $(\vec{e})^{-1}$ inducing an oriented 2-cycle $\vec{C}_{2}$. Then, fastening $\vec{e}$ and $(\vec{e})^{-1}$ allows to obtain precisely the edge $e$ in the graph $G$. Is it possible to orient all shortest cycles in a distance-transitive graph $G$ so that each two O-O $(k-1)$-arcs of $G$ are in just two oriented shortest cycles, where $k=$ largest $\ell$ such that $G$ is $\ell$-arc transitive? It is shown below that this is so just for 7 of the 12 cubic distance-transitive graphs $G$, leading to 7 corresponding minimum connected digraphs $\mathcal{S}(G)$ in which all oriented shortest cycles of $G$ are "pulled apart" by means of a graph-theoretical operation explained in Section 4 below.

Given a collection $\mathcal{C}$ of (di)graphs closed under isomorphisms, a (di)graph $G$ is said to be $\mathcal{C}$-ultrahomogeneous (or $\mathcal{C}$-UH) if every isomorphism between two induced members of $\mathcal{C}$ in $G$ extends to an automorphism of $G$. If $\mathcal{C}$ is the isomorphism class of a (di)graph $H$, we say that such a $G$ is $\{H\}-\mathrm{UH}$ or $H$-UH. In [14], $\mathcal{C}$-UH graphs are defined and studied when $\mathcal{C}$ is the collection of either the complete graphs, or the disjoint unions of complete graphs, or the complements of those unions.

Let $M$ be an induced subgraph of a graph $H$ and let $G$ be both an $M$-UH and an $H$-UH graph. We say that $G$ is an $\{H\}_{M}-U H$ graph if, for each induced copy $H_{0}$ of $H$ in $G$ and for each induced copy $M_{0}$ of $M$ in $H_{0}$, there exists exactly one induced copy $H_{1} \neq H_{0}$ of $H$ in $G$ with $V\left(H_{0}\right) \cap V\left(H_{1}\right)=V\left(M_{0}\right)$ and $E\left(H_{0}\right) \cap E\left(H_{1}\right)=E\left(M_{0}\right)$. The vertex and edge conditions above can be condensed as $H_{0} \cap H_{1}=M_{0}$. We say that such a $G$ is tightly fastened. This is generalized by saying that an $\{H\}_{M}-\mathrm{UH}$ graph $G$ is an $\ell$-fastened $\{H\}_{M}$-UH graph if given an induced copy $H_{0}$ of $H$ in $G$ and an induced copy $M_{0}$ of $M$ in $H_{0}$, then there exist exactly $\ell$ induced copies $H_{i} \neq H_{0}$ of $H$ in $G$ such that $H_{i} \cap H_{0} \supseteq M_{0}$, for each $i=1,2, \ldots, \ell$, with at least $H_{1} \cap H_{0}=M_{0}$.

Let $\vec{M}$ be an induced subdigraph of a digraph $\vec{H}$ and let the graph $G$ be both an $\vec{M}$-UH and an $\vec{H}$-UH digraph. We say that $G$ is an $\{\vec{H}\}_{\vec{M}}$-UH digraph if for each induced copy $\vec{H}_{0}$ of $\vec{H}$ in $\vec{G}$ and for each induced copy $\vec{M}_{0}$ of $\vec{M}$ in $\vec{H}_{0}$ there exists exactly one induced copy 
$\vec{H}_{1} \neq \vec{H}_{0}$ of $\vec{H}$ in $G$ with $V\left(\vec{H}_{0}\right) \cap V\left(\vec{H}_{1}\right)=V\left(\vec{M}_{0}\right)$ and $A\left(\vec{H}_{0}\right) \cap \bar{A}\left(\vec{H}_{1}\right)=A\left(\vec{M}_{0}\right)$, where $\bar{A}\left(\vec{H}_{1}\right)$ is formed by those arcs $(\vec{e})^{-1}$ whose orientations are reversed with respect to the orientations of the arcs $\vec{e}$ of $A\left(\vec{H}_{1}\right)$. Again, we say that such a $G$ is tightly fastened. This case is used in the constructions of Section 4.

Given a finite graph $H$ and a subgraph $M$ of $H$ with $|V(H)|>3$, we say that a graph $G$ is (strongly fastened) $S F\{H\}_{M}$-UH if there is a descending sequence of connected subgraphs $M=M_{1}, M_{2} \ldots, M_{t} \equiv K_{2}$ such that: (a) $M_{i+1}$ is obtained from $M_{i}$ by the deletion of a vertex, for $i=1, \ldots, t-1$ and (b) $G$ is a $\left(2^{i}-1\right)$-fastened $\{H\}_{M_{i}}$-UH graph, for $i=1, \ldots, t$.

This paper deals with the above defined $\mathcal{C}$-UH concepts applied to cubic distance-transitive (CDT) graphs [3]. A list of them and their main parameters follows:

\begin{tabular}{|l|l|l|l|l|l|l|l|l|l|}
\hline CDT graph $G$ & $n$ & $d$ & $g$ & $k$ & $\eta$ & $a$ & $b$ & $h$ & $\kappa$ \\
\hline Tetrahedral graph $K_{4}$ & 4 & 1 & 3 & 2 & 4 & 24 & 0 & 1 & 1 \\
Thomsen graph $K_{3,3}$ & 6 & 2 & 4 & 3 & 9 & 72 & 1 & 1 & 2 \\
3-cube graph $Q_{3}$ & 8 & 3 & 4 & 2 & 6 & 48 & 1 & 1 & 1 \\
Petersen graph & 10 & 2 & 5 & 3 & 12 & 120 & 0 & 0 & 0 \\
Heawood graph & 14 & 3 & 6 & 4 & 28 & 336 & 1 & 1 & 0 \\
Pappus graph & 18 & 4 & 6 & 3 & 18 & 216 & 1 & 1 & 0 \\
Dodecahedral graph & 20 & 5 & 5 & 2 & 12 & 120 & 0 & 1 & 1 \\
Desargues graph & 20 & 5 & 6 & 3 & 20 & 240 & 1 & 1 & 3 \\
Coxeter graph & 28 & 4 & 7 & 3 & 24 & 336 & 0 & 0 & 3 \\
Tutte 8-cage & 30 & 4 & 8 & 5 & 90 & 1440 & 1 & 1 & 2 \\
Foster graph & 90 & 8 & 10 & 5 & 216 & 4320 & 1 & 1 & 0 \\
Biggs-Smith graph & 102 & 7 & 9 & 4 & 136 & 2448 & 0 & 1 & 0 \\
\hline
\end{tabular}

where $n=$ order; $d=$ diameter; $g=$ girth; $k=$ AT or arc-transitivity (= largest $\ell$ such that $G$ is $\ell$-arc transitive); $\eta=$ number of $g$-cycles; $a=$ number of automorphisms; $b$ (resp. $h$ ) $=1$ if $G$ is bipartite (resp. hamiltonian) and $=0$ otherwise; and $\kappa$ is defined as follows: let $P_{k}$ and $\vec{P}_{k}$ be respectively a $(k-1)$-path and a directed $(k-1)$-path (of length $k-1$ ); let $C_{g}$ and $\vec{C}_{g}$ be respectively a cycle and a directed cycle of length $g$; then (see Theorem 3 below): $\kappa=0$, if $G$ is not $\left(\vec{C}_{g} ; \vec{P}_{k}\right)-\mathrm{UH} ; \kappa=1$, if $G$ is planar; $\kappa=2$, if $G$ is $\left\{\overrightarrow{C_{g}}\right\}_{\vec{P}_{k}}-\mathrm{UH}$ with $g=2(k-1) ; \kappa=3$, if $G$ is $\left\{\overrightarrow{C_{g}}\right\}_{\vec{P}_{k}}$-UH with $g>2(k-1)$.

In Section 3 below, Theorem 2 proves that every CDT graph is an SF $\left\{C_{g}\right\}_{P_{k}}$-UH graph, while Theorem 3 establishes exactly which CDT graphs are not $\left\{\vec{C}_{g}\right\}_{\vec{P}_{k}}$-UH digraphs; in fact 5 of them. Section 4 shows that each of the remaining 7 CDT graphs $G$ yields a digraph $\mathcal{S}(G)$ whose vertices are the $(k-1)$-arcs of $G$, an arc in $\mathcal{S}(G)$ between each two vertices representing corresponding $(k-1)$-arcs in a common oriented $g$-cycle of $G$ and sharing just one $(k-2)$-arc; additional arcs of $\mathcal{S}(G)$ appearing in O-O pairs associated with the reversals of $(k-1)$-arcs of $G$. Moreover, Theorem 4 asserts that each $\mathcal{S}(G)$ is as claimed and itemized at end of the Introduction above.

\section{$3\left(C_{g}, P_{k}\right)$-UH properties of CDT graphs}

Theorem 3.1. Let $G$ be a CDT graph of girth $=g$, AT $=k$ and order $=n$. Then, $G$ is an $\mathrm{SF}\left\{C_{g}\right\}_{P_{k}}$-UH graph. In particular, $G$ has exactly $2^{k-2} 3 n g^{-1} g$-cycles. 
Proof. We have to see that each CDT graph $G$ with girth $=g$ and AT $=k$ is a $\left(2^{i+1}-1\right)$ fastened $\left\{C_{g}\right\}_{P_{k-i}}$-UH graph, for $i=0,1, \ldots, k-2$. In fact, each $(k-i-1)$-path $P=P_{k-i}$ of any such $G$ is shared by exactly $2^{i+1} g$-cycles of $G$, for $i=0,1, \ldots, k-2$. For example if $k=4$, then any edge (resp. 2-path, resp. 3-path) of $G$ is shared by 8 (resp. 4 , resp. 2) $g$-cycles of $G$. This means that a $g$-cycle $C_{g}$ of $G$ shares a $P_{2}$ (resp. $P_{3}$, resp. $P_{4}$ ) with exactly other 7 (resp. 3, resp. 1) $g$-cycles. Thus $G$ is an SF $\left\{C_{g}\right\}_{P_{i+2}}$-UH graph, for $i=0,1, \ldots, k-2$. The rest of the proof depends on the particular cases analyzed in the proof of Theorem 3 below and on some simple counting arguments for the pertaining numbers of $g$-cycles.

Given a CDT graph $G$, there are just two $g$-cycles shared by each $(k-1)$-path. If in addition $G$ is a $\left\{\vec{C}_{g}\right\}_{\vec{P}_{k}}$-UH graph, then there exists an assignment of an orientation for each $g$-cycle of $G$, so that the two $g$-cycles shared by each $(k-1)$-path receive opposite orientations. We say that such an assignment is a $\left\{\vec{C}_{g}\right\}_{\vec{P}_{k}}-O-O$ assignment (or $\left\{\vec{C}_{g}\right\}_{\vec{P}_{k}}$-OOA). The collection of $\eta$ oriented $g$-cycles corresponding to the $\eta g$-cycles of $G$, for a particular $\left\{\vec{C}_{g}\right\}_{\vec{P}_{k}}$-OOA will be called an $\left\{\eta \vec{C}_{g}\right\}_{\vec{P}_{k}}$-OOC. Each such $g$-cycle will be expressed with its successive composing vertices expressed between parentheses but without separating commas, (as is the case for arcs $u v$ and 2-arcs $u v w$ ), where as usual the vertex that succeeds the last vertex of the cycle is its first vertex.

Theorem 3.2. The CDT graphs $G$ of girth $=g$ and $\mathrm{AT}=k$ that are not $\left\{\vec{C}_{g}\right\}_{\overrightarrow{P_{k}}}-\mathrm{UH}$ digraphs are the graphs of Petersen, Heawood, Pappus, Foster and Biggs-Smith. The remaining 7 CDT graphs are $\left\{\overrightarrow{C_{g}}\right\}_{\overrightarrow{P_{k}}}$-UH digraphs.

Proof. Let us consider the case of each CDT graph sequentially. The graph $K_{4}$ on vertex set $\{1,2,3,0\}$ admits the $\left\{4 \vec{C}_{3}\right\}_{\vec{P}_{2}}$-OOC $\{(123),(210),(301),(032)\}$. The graph $K_{3,3}$ obtained from $K_{6}$ (with vertex set $\{1,2,3,4,5,0\}$ ) by deleting the edges of the triangles $(1,3,5)$ and $(2,4,0)$ admits the $\left\{9 \vec{C}_{4}\right\}_{\vec{P}_{3}}$-OOC $\{(1234),(3210),(4325),(1430),(2145)$, $(0125),(5230),(0345),(5410)\}$. The graph $Q_{3}$ with vertex set $\{0, \ldots, 7\}$ and edge set $\{01,23,45,67,02,13,46,57,04,15,26,37\}$ admits the $\left\{6 \vec{C}_{4}\right\}_{\vec{P}_{2}}$-OOC $\{(0132),(1045)$, (3157), (2376), (0264), (4675)\}.

The Petersen graph Pet is obtained from the disjoint union of the 5-cycles $\mu^{\infty}=\left(u_{0} u_{1} u_{2}\right.$ $\left.u_{3} u_{4}\right)$ and $\nu^{\infty}=\left(v_{0} v_{2} v_{4} v_{1} v_{3}\right)$ by the addition of the edges $\left(u_{x}, v_{x}\right)$, for $x \in \mathbf{Z}_{5}$. Apart from the two 5-cycles given above, the other 105 -cycles of Pet can be denoted by $\mu^{x}=$ $\left(u_{x-1} u_{x} u_{x+1} v_{x+1} v_{x-1}\right)$ and $\nu^{x}=\left(v_{x-2} v_{x} v_{x+2} u_{x+2} u_{x-2}\right)$, for each $x \in \mathbf{Z}_{5}$. Then, the following sequence of alternating 5-cycles and 2-arcs starts and ends up with opposite orientations:

$$
\mu_{-}^{2} \quad u_{3} u_{2} u_{1} \mu_{+}^{\infty} u_{0} u_{1} u_{2} \quad \mu_{-}^{1} u_{2} v_{2} v_{0} \quad \nu_{-}^{0} v_{3} u_{3} u_{2} \quad \mu_{+}^{2}
$$

where the subindexes \pm indicate either a forward or backward selection of orientation and each 2-path is presented with the orientation of the previously cited 5-cycle but must be present in the next 5-cycle with its orientation reversed. Thus Pet cannot be a $\left\{\vec{C}_{5}\right\}_{\vec{P}_{3}}-\mathrm{UH}$ digraph.

Another way to see this is via the auxiliary table indicated below, that presents the form in which the 5-cycles above share the vertex sets of 2-arcs, either $\mathrm{O}-\mathrm{O}$ or not. The table details, for each one of the 5-cycles $\xi=\mu^{\infty}, \nu^{\infty}, \mu^{0}, \nu^{0}$, (expressed as $\xi=\left(\xi_{0}, \ldots, \xi_{4}\right)$ in 
the shown vertex notation), each 5-cycle $\eta$ in $\left\{\mu^{i}, \nu^{i} ; i=\infty, 0, \ldots, 4\right\} \backslash\{\xi\}$ that intersects $\xi$ in the succeeding 2-paths $\xi_{i} \xi_{i+1} \xi_{i+2}$, for $i=0, \ldots, 4$, with additions involving $i$ taken mod 5. Each such $\eta$ in the auxiliary table has either a preceding minus sign, if the corresponding 2 -arcs in $\xi$ and $\eta$ are $\mathrm{O}-\mathrm{O}$, or a plus sign, otherwise. Each $-\eta_{j}$ (resp. $\eta_{j}$ ) shown in the table has the subindex $j$ indicating the equality of initial vertices $\eta_{j}=\xi_{i+2}$ (resp. $\eta_{j}=\xi_{i}$ ) of those 2-arcs, for $i=0, \ldots, 4$ :

$$
\begin{array}{ll}
\mu^{\infty}:\left(+\mu_{0}^{1},+\mu_{0}^{2},+\mu_{0}^{3},+\mu_{0}^{4},+\mu_{0}^{0}\right), & \nu^{\infty}:\left(+\nu_{0}^{2},+\nu_{0}^{4},+\nu_{0}^{1},+\nu_{0}^{3},+\nu_{0}^{0}\right), \\
\mu^{0}:\left(+\mu_{4}^{\infty},+\nu_{3}^{3},-\nu_{1}^{4},-\nu_{4}^{1},+\nu_{2}^{2}\right), & \nu^{0}:\left(+\nu_{4}^{\infty},-\mu_{2}^{1},+\mu_{4}^{3},+\mu_{1}^{2},-\mu_{3}^{4}\right) .
\end{array}
$$

This partial auxiliary table is extended to the whole auxiliary table by adding $x \in \mathbf{Z}_{4}$ uniformly mod 5 to all superindexes $\neq \infty$, reconfirming that Pet is not $\left\{\vec{C}_{5}\right\}_{\vec{P}_{3}}-\mathrm{UH}$.

For each positive integer $n$, let $I_{n}$ stand for the $n$-vertex cycle $(0,1, \ldots, n-1)$. The Heawood graph $\mathrm{Hea}$ is obtained from $I_{14}$ by adding the edges $(2 x, 5+2 x)$, where $x \in$ $\{1, \ldots, 7\}$ and operations are in $\mathbf{Z}_{14}$. The 28 6-cycles of $\mathrm{Hea}$ include the following 7 6-cycles:

$$
\begin{array}{ll}
\gamma^{x}=(2 x, 2 x+1,2 x+2,2 x+3,2 x+4,2 x+5), & \delta^{x}=(2 x \quad, 2 x+5,2 x+6,2 x+7,2 x+8,2 x+13), \\
\epsilon^{x}=(2 x, 2 x+5,2 x+4,2 x+9,2 x+8,2 x+13), & \zeta^{x}=(2 x+12,2 x+3,2 x+4,2 x+5,2 x \quad, 2 x+13),
\end{array}
$$

where $x \in \mathbf{Z}_{7}$. Now, the following sequence of alternating 6-cycles and 3-arcs starts and ends with opposite orientations for $\gamma_{0}$ :

$$
\begin{array}{llllllllllllllll}
\gamma_{+}^{0} & 2345 & \gamma_{-}^{1} & 7654 & \gamma_{+}^{2} & 6789 & \gamma_{-}^{3} & b a 98 & \gamma_{+}^{4} & \text { abcd } & \gamma_{-}^{5} & 10 d c & \gamma_{+}^{6} & 0123 & \gamma_{-}^{0},
\end{array}
$$

(where tridecimal notation is used, up to $d=13$ ). Thus $H e a$ cannot be a $\left\{\vec{C}_{7}\right\}_{\vec{P}_{4}}$-UH digraph. Another way to see this is via an auxiliary table for Hea obtained in a fashion similar to that of the one for Pet above from:

$$
\begin{array}{ll}
\gamma^{0}:\left(+\gamma_{2}^{6},+\delta_{1}^{5},+\gamma_{0}^{1},+\zeta_{1}^{6},-\epsilon_{1}^{5},-\zeta_{4}^{0}\right) ; & \epsilon^{0}:\left(+\epsilon_{2}^{5},-\gamma_{4}^{2},+\epsilon_{0}^{2},+\zeta_{5}^{4},+\delta_{4}^{0},-\zeta_{2}^{6}\right) ; \\
\delta^{0}:\left(+\zeta_{0}^{0},+\gamma_{1}^{2},-\zeta_{3}^{3},+\delta_{5}^{4},+\epsilon_{4}^{0},+\delta_{3}^{3}\right) ; & \zeta^{0}:\left(+\delta_{0}^{0},+\gamma_{3}^{1},-\epsilon_{5}^{1},-\delta_{2}^{4},-\gamma_{5}^{0},+\epsilon_{3}^{3}\right) .
\end{array}
$$

This reaffirms that Hea is not $\left\{\vec{C}_{6}\right\}_{\vec{P}_{4}}-\mathrm{UH}$.

The Pappus graph Pap is obtained from $I_{18}$ by adding to it the edges $(1+6 x, 6+6 x),(2+$ $6 x, 9+6 x),(4+6 x, 11+6 x)$, for $x \in\{0,1,2\}$, with sums and products taken mod 18 . The 6-cycles of Pap are expressible as: $A_{0}=(123456), B_{0}=(3210 d e), C_{0}=(34 b c d e)$, $D_{0}=(165 g h 0), E_{0}=(329 a b 4)$ (where octodecimal notation is used, up to $h=17$ ), the 6-cycles $A_{x}, B_{x}, C_{x}, D_{x}, E_{x}$ obtained by uniformly adding $6 x \bmod 18$ to the vertices of $A_{0}, B_{0}, C_{0}, D_{0}, E_{0}$, for $x \in \mathbf{Z}_{3} \backslash\{0\}$, and $F_{0}=(3298 f e), F_{1}=(h g 54 b a), F_{2}=$ $(167 c d 0)$. No orientation assignment makes these cycles into an $\left\{18 \vec{C}_{6}\right\}_{\vec{P}_{3}}$-OOC, for the following sequence of alternating 6-cycles and 2-arcs (with orientation reversed between each preceding 6-cycle to corresponding succeeding 6-cycle) reverses the orientation of its initial 6-cycle in its terminal one:

$$
\begin{aligned}
& D_{1}^{-1} 654 A_{0} 123 B_{0} 210 C_{1} h 01 D_{0}^{-1} g 56 C_{2}^{-1} 876 B_{1}^{-1} 789 A_{1}^{-1} c b a D_{2} a b c A_{1}^{-1} 987 B_{1}^{-1} 678 C_{2}^{-1} 765 D_{1} \\
&=(654 b c 7) 654(123456) 123(3210 d e) 210(0129 a h) h 01(10 h g 56) g 56(5 g f 876) 876 \\
&(216789) 789(c b a 987) c b a(d 0 h a b c) a b c(c b a 987) 987(216789) 678(5 g f 876) 765(c b 4567) .
\end{aligned}
$$

Another way to see this is via an auxiliary table for Pap obtained in a fashion similar to those above for Pet and Hea, where $x=0,1,2(\bmod 3)$ :

$$
\begin{array}{lll|l}
-A_{x}:\left(B_{x}, E_{x}, E_{x+2}, D_{x+1}, D_{x}\right. & \left., B_{x+1}\right) ; & -F_{0}:\left(E_{0}, B_{1}, E_{1}, B_{2}, E_{2}, B_{0}\right) ; \\
-B_{x}:\left(A_{x}, C_{x+1}, F_{2}, A_{x+2}, C_{x}\right. & \left., F_{0}\right) ; & -F_{1}:\left(D_{0}, E_{2}, D_{1}, E_{0}, D_{2}, E_{1}\right) ; \\
-C_{x}:\left(E_{x}, D_{x+1}, D_{x+2}, B_{x+2}, B_{x}\right. & \left., E_{x+2}\right) ; & -F_{2}:\left(B_{1}, D_{1}, B_{2}, D_{2}, B_{0}, D_{0}\right) ; \\
-D_{x}:\left(A_{x}, C_{x+2}, F_{1}, A_{x+2}, C_{x+1}, F_{2}\right) ; & \\
-E_{x}:\left(F_{0}, C_{x+1}, A_{x+1}, F_{1}, C_{x}, A_{x}\right) &
\end{array}
$$


This reaffirms that Pap is not a $\left\{\vec{C}_{6}\right\}_{\vec{P}_{3}}$-UH digraph. In fact, observe that any two 6-cycles here that share a 2-path possess the same orientation, in total contrast to what happens in the 7 cases that are being shown to be $\left\{\vec{C}_{g}\right\}_{\vec{P}_{k}}$-UH digraphs, in the course of this proof.

The Desargues graph Des is obtained from the 20-cycle $I_{20}$, with vertices $4 x, 4 x+1,4 x+$ $2,4 x+3$ redenoted alternatively $x_{0}, x_{1}, x_{2}, x_{3}$, respectively, for $x \in \mathbf{Z}_{5}$, by adding the edges $\left(x_{3},(x+2)_{0}\right)$ and $\left(x_{1},(x+2)_{2}\right)$, where operations are mod 5. Then, Des admits a $\left\{20 \vec{C}_{6}\right\}_{\vec{P}_{3}}$-OOC formed by the oriented 6-cycles $A^{x}, B^{x}, C^{x}, D^{x}$, for $x \in\{0, \ldots, 4\}$, where

$$
\begin{array}{ll}
A^{x}=\left(x_{0} x_{1} x_{2} x_{3}(x+1)_{0}(x+4)_{3}\right), & B^{x}=\left(x_{1} x_{0}(x+4)_{3}(x+4)_{2}(x+2)_{1}(x+2)_{2}\right), \\
C^{x}=\left(x_{2} x_{1} x_{0}(x+3)_{3}(x+3)_{2}(x+3)_{1}\right), & D^{x}=\left(x_{0}(x+4)_{3}(x+1)_{0}(x+1)_{1}(x+3)_{2}(x+3)_{3}\right) .
\end{array}
$$

The successive copies of $\vec{P}_{3}$ here, when reversed in each case, must belong to the following remaining oriented 6-cycles:

$$
\begin{array}{ll}
A^{x}:\left(C^{x}, C^{x+2}, B^{x+1}, D^{x+1}, D^{x}, B^{x}\right) ; & B^{x}:\left(A^{x}, A^{x+4}, D^{x+1}, C^{x+4}, C^{x+2}, D^{x+4}\right) ; \\
C^{x}:\left(A^{x}, D^{x+4}, D^{x}, A^{x+3}, B^{x+1}, B^{x+3}\right) ; & D^{x}:\left(A^{x}, C^{x+1}, B^{x+1}, B^{x+4}, C^{x}, A^{x+4}\right) ;
\end{array}
$$

showing that they constitute effectively an $\left\{\eta \vec{C}_{g}\right\}_{\vec{P}_{k}}$-OOC.

The dodecahedral graph $\Delta$ is a 2-covering graph of the Petersen graph $H$, where each vertex $u_{x}$, (resp., $\left.v_{x}\right)$, of $H$ is covered by two vertices $a_{x}, c_{x}$, (resp. $\left.b_{x}, d_{x}\right)$. A $\left\{12 \vec{C}_{5}\right\}_{\vec{P}_{2}}{ }^{-}$ OOC of $\Delta$ is given by the oriented 5-cycles $\left(a_{0} a_{1} a_{2} a_{3} a_{4}\right),\left(c_{4} c_{3} c_{2} c_{1} c_{0}\right)$ and, for each $x \in \mathbf{Z}_{5}$, also by $\left(a_{x} d_{x} b_{x-2} d_{x+1} a_{x+1}\right)$ and $\left(d_{x} b_{x+2} c_{x+2} c_{x-2} b_{x-2}\right)$.

The Tutte 8-cage Tut is obtained from $I_{30}$, with vertices $6 x, 6 x+1,6 x+2,6 x+3,6 x+$ $4,6 x+5$ denoted alternatively $x_{0}, x_{1}, x_{2}, x_{3}, x_{4}, x_{5}$, respectively, for $x \in \mathbf{Z}_{5}$, by adding the edges $\left(x_{5},(x+2)_{0}\right),\left(x_{1},(x+1)_{4}\right)$ and $\left(x_{2},(x+2)_{3}\right)$. Then, Tut admits the $\left\{90 \vec{C}_{8}\right\}_{\vec{P}_{5}}{ }^{-}$ OOC formed by the oriented 8-cycles:

$$
\begin{array}{lll}
A^{0}=\left(4_{5} 0_{0} 0_{1} 0_{2} 0_{3} 0_{4} 0_{5} 1_{0}\right), & B^{0}=\left(4_{2} 4_{3} 4_{4} 4_{5} 1_{0} 1_{1} 1_{2} 1_{3}\right), & C^{0}=\left(0_{2} 0_{3} 0_{4} 4_{1} 4_{0} 2_{5} 2_{4} 2_{3}\right), \\
D^{0}=\left(3_{3} 3_{2} 3_{1} 4_{4} 4_{3} 4_{2} 1_{3} 1_{2}\right), & E^{0}=\left(4_{5} 1_{0} 0_{5} 0_{4} 4_{1} 4_{0} 3_{5} 0_{0}\right), & F^{0}=\left(4_{5} 0_{0} 3_{5} 4_{0} 2_{5} 2_{4} 1_{1} 1_{0}\right), \\
G^{0}=\left(1_{0} 1_{1} 2_{4} 2_{3} 0_{2} 0_{1} 0_{0} 4_{5}\right), & H^{0}=\left(2_{3} 2_{4} 1_{1} 1_{0} 0_{5} 0_{4} 0_{3} 0_{2}\right), & I^{0}=\left(0_{1} 0_{2} 0_{3} 0_{4} 4_{1} 4_{2} 1_{3} 1_{4}\right), \\
J^{0}=\left(1_{0} 0_{5} 0_{4} 0_{3} 3_{2} 3_{1} 4_{4} 4_{5}\right), & K^{0}=\left(3_{1} 3_{2} 0_{3} 0_{2} 0_{1} 0_{0} 4_{5} 4_{4}\right), & L^{0}=\left(2_{3} 2_{4} 2_{5} 3_{0} 3_{1} 3_{2} 0_{3} 0_{2}\right), \\
M^{0}=\left(3_{5} 4_{0} 4_{1} 0_{4} 0_{3} 0_{2} 0_{1} 0_{0}\right), & N^{0}=\left(0_{0} 0_{1} 1_{4} 1_{5} 2_{0} 2_{1} 3_{4} 3_{5}\right), & O^{0}=\left(4_{2} 4_{3} 2_{2} 2_{1} 3_{4} 3_{3} 1_{2} 1_{3}\right), \\
P^{0}=\left(4_{5} 4_{4} 4_{3} 4_{2} 4_{1} 0_{4} 0_{5} 1_{0}\right), & Q^{0}=\left(4_{0} 4_{1} 4_{2} 1_{3} 1_{4} 1_{5} 3_{0} 2_{5}\right), & R^{0}=\left(0_{1} 0_{2} 0_{3} 3_{2} 3_{1} 3_{0} 1_{5} 1_{4}\right),
\end{array}
$$

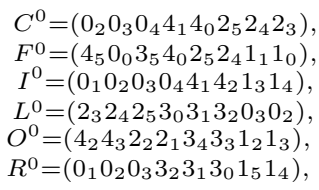

together with those obtained by adding $y \in \mathbf{Z}_{5}$ uniformly mod 5 to all numbers $x$ of vertices $x_{i}$ in $A^{0}, \ldots, R^{0}$, for each $y=1,2,3,4$, yielding in each case oriented 8 -cycles $A^{y}, \ldots, R^{y}$.

The Coxeter graph Cox is obtained from three 7-cycles $\left(u_{1} u_{2} u_{3} u_{4} u_{5} u_{6} u_{0}\right),\left(v_{4} v_{6} v_{1} v_{3} v_{5}\right.$ $\left.v_{0} v_{2}\right),\left(t_{3} t_{6} t_{2} t_{5} t_{1} t_{4} t_{0}\right)$ by adding a copy of $K_{1,3}$ with degree-1 vertices $u_{x}, v_{x}, t_{x}$ and a central degree-3 vertex $z_{x}$, for each $x \in \mathbf{Z}_{7}$. Cox admits the $\left\{24 \vec{C}_{7}\right\}_{\vec{P}_{3}}$-OOC:

$$
\begin{aligned}
& \left\{0^{1}=\left(u_{1} u_{2} u_{3} u_{4} u_{5} u_{6} u_{0}\right)\right. \text {, }
\end{aligned}
$$

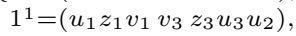

$$
\begin{aligned}
& 2^{1}=\left(\begin{array}{llll}
v_{5} z_{5} u_{5} u_{4} u_{3} & z_{3} & v_{3}
\end{array}\right), \\
& 3^{1}=\left(\begin{array}{llll}
v_{5} & v_{0} z_{0} u_{0} u_{6} & u_{5} & z_{5}
\end{array}\right), \\
& 4^{1}=\left(u_{1} u_{0} z_{0} v_{0} v_{2} z_{2} u_{2}\right) \text {, } \\
& 5^{1}=\left(\begin{array}{llll}
z_{4} u_{4} & u_{3} u_{2} z_{2} & v_{2} & v_{4}
\end{array}\right) \text {, } \\
& 6^{1}=\left(\begin{array}{llll}
z_{4} v_{4} v_{6} z_{6} u_{6} & u_{5} & u_{4}
\end{array}\right),
\end{aligned}
$$

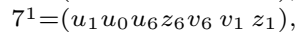

$$
\begin{aligned}
& 0^{3}=\left(t_{1} t_{5} t_{2} t_{6} t_{3} t_{0} t_{4}\right), \\
& 1^{3}=\left(t_{6} t_{2} t_{5} z_{5} u_{5} u_{6} z_{6}\right), \\
& 2^{3}=\left(u_{1} z_{1} t_{1} t_{4} t_{0} z_{0} u_{0}\right), \\
& 3^{3}=\left(t_{6} t_{2} z_{2} u_{2} u_{3} z_{3} t_{3}\right), \\
& 4^{3}=\left(z_{4} u_{4} u_{5} z_{5} t_{5} t_{1} t_{4}\right), \\
& 5^{3}=\left(t_{6} z_{6} u_{6} u_{0} z_{0} t_{0} t_{3}\right), \\
& 6^{3}=\left(u_{1} u_{2} z_{2} t_{2} t_{5} t_{1} z_{1}\right), \\
& \left.7^{3}=\left(z_{4} t_{4} t_{0} t_{3} z_{3} u_{3} u_{4}\right)\right\}
\end{aligned}
$$


The Foster graph Fos is obtained from $I_{90}$, with vertices $6 x, 6 x+1,6 x+2,6 x+3,6 x+$ $4,6 x+5$ denoted alternatively $x_{0}, x_{1}, x_{2}, x_{3}, x_{4}, x_{5}$, respectively, for $x \in \mathbf{Z}_{15}$, by adding the edges $\left(x_{4},(x+2)_{1}\right),\left(x_{0},(x+2)_{5}\right)$ and $\left(x_{2},(x+6)_{3}\right)$. The 21610 -cycles of Fos include the following 1510 -cycles, where $x \in \mathbf{Z}_{15}$ :

$$
\phi^{x}=\left(x_{4} x_{5}(x+1)_{0}(x+1)_{1}(x+1)_{2}(x+1)_{3}(x+1)_{4}(x+1)_{5}(x+2)_{0}(x+2)_{1}\right) .
$$

Then, the following sequence of alternating 10-cycles and 4-arcs:

$$
\phi_{+}^{0}\left[1_{4}\right] \phi_{-}^{1}\left[3_{1}\right] \phi_{+}^{2}\left[3_{4}\right] \phi_{-}^{3}\left[5_{1}\right] \phi_{+}^{4}\left[5_{4}\right] \phi_{-}^{5}\left[7_{1}\right] \phi_{+}^{6}\left[7_{4}\right] \phi_{-}^{7}\left[9_{1}\right] \phi_{+}^{8}\left[9_{4}\right] \phi_{-}^{9}\left[b_{1}\right] \phi_{+}^{a}\left[b_{4}\right] \phi_{-}^{b}\left[d_{1}\right] \phi_{+}^{c}\left[d_{4}\right] \phi_{-}^{d}\left[0_{1}\right] \phi_{+}^{e}\left[0_{4}\right]
$$

may be continued with $\phi_{-}^{0}$, with opposite orientation to that of the initial $\phi_{+}^{0}$, where $\left[x_{j}\right]$ stands for a 3 -arc starting at the vertex $x_{j}$ in the previously cited (to the left) oriented 10cycle. Thus Fos cannot be a $\left\{\vec{C}_{10}\right\}_{\vec{P}_{5}}$-UH digraph. Another way to see this is via the following table of 10 -cycles of Fos, where the 10 -cycle $\phi_{0}$ intervenes as 10 -cycle $0^{0}$ :

$$
\begin{array}{ll}
0^{0}=\left(0_{4} 0_{5} 1_{0} 1_{1} 1_{2} 1_{3} 1_{4} 1_{5} 2_{0} 2_{1}\right) & 8^{0}=\left(0_{2} 0_{3} 9_{2} 9_{1} 7_{4} 7_{5} 8_{0} 8_{1} 6_{4} 6_{3}\right) \\
1^{0}=\left(0_{0} 0_{1} 0_{2} 0_{3} 0_{4} 2_{1} 2_{2} 2_{3} 2_{4} 2_{5}\right) & 9^{0}=\left(0_{4} 0_{5} d_{0} d_{1} d_{2} 4_{3} 4_{4} 4_{5} 2_{0} 2_{1}\right) \\
2^{0}=\left(0_{3} 0_{4} 0_{5} 1_{0} 1_{1} 1_{2} 7_{3} 7_{4} 9_{1} 9_{2}\right) & a^{0}=\left(0_{4} 0_{5} d_{0} d_{1} b_{4} b_{3} b_{2} 2_{3} 2_{2} 2_{1}\right) \\
3^{0}=\left(0_{3} 0_{4} 0_{5} d_{0} c_{5} a_{0} 9_{5} 9_{4} 9_{3} 9_{2}\right) & b^{0}=\left(0_{4} 0_{5} 1_{0} 3_{5} 3_{4} 5_{1} 5_{0} 4_{5} 2_{0} 2_{1}\right) \\
4^{0}=\left(0_{0} 0_{1} 0_{2} 6_{3} 6_{2} 6_{1} 6_{0} 5_{5} 3_{0} 2_{5}\right) & c^{0}=\left(0_{2} 0_{3} 0_{4} 2_{1} 2_{2} 8_{3} 8_{2} 8_{1} 6_{4} 6_{3}\right) \\
5^{0}=\left(0_{4} 0_{5} 1_{0} 3_{5} 4_{0} 4_{1} 2_{4} 2_{3} 2_{2} 2_{1}\right) & d^{0}=\left(0_{3} 0_{4} 2_{1} 2_{0} 4_{5} 5_{0} 7_{5} 7_{4} 9_{1} 9_{2}\right) \\
6^{0}=\left(0_{3} 0_{4} 0_{5} 1_{0} 3_{5} 3_{4} 3_{3} 3_{2} 9_{3} 9_{2}\right) & e^{0}=\left(0_{5} 1_{0} 3_{5} 4_{0} 6_{5} 7_{0} 9_{5} a_{0} c_{5} d_{0}\right) \\
7^{0}=\left(0_{0} 0_{1} R_{2} 0_{3} 9_{2} 9_{3} 3_{2} 3_{1} 3_{0} 2_{5}\right) & f^{0}=\left(0_{2} 0_{3} 9_{2} 9_{3} 3_{2} 3_{3} c_{2} c_{3} 6_{2} 6_{3}\right)
\end{array}
$$

where (a) hexadecimal notation of integers is used; (b) the first 1410 -cycles $x^{0},(x=$ $0, \ldots, 13=d)$, yield corresponding 10 -cycles $x^{j},\left(j \in \mathbf{Z}_{15}\right)$. via translation modulo 15 of all indexes; and (c) the last two cycles, $e^{0}$ and $f^{0}$, yield merely additional 10-cycles $e^{1}, e^{2}, f^{1}$ and $f^{2}$ by the same index translation. A corresponding auxiliary table as in the discussions for Pet, Hea and Pap above, in which the \pm assignments are missing and left as an exercise for the reader is as follows:

$$
\begin{array}{ll}
0^{0}:\left(2^{0}, 4^{a}, 1^{1}, 1^{e}, 3^{7}, 2^{1}, 8^{9}, b^{c}, b^{0}, 8^{8}\right) & 8^{0}:\left(c^{7}, d^{0}, 5^{7}, 0^{7}, 0^{6}, b^{4}, d^{0}, c^{0}, 6^{6}, 7^{6}\right) \\
1^{0}:\left(0^{e}, 5^{d}, c^{0}, c^{9}, 5^{0}, 0^{1}, 6^{e}, 9^{d}, 9^{2}, 7^{0}\right) & 9^{0}:\left(1^{d}, 4^{d}, 2^{c}, 2^{4}, 3^{4}, 1^{2}, a^{4}, b^{0}, b^{c}, a^{0}\right) \\
2^{0}:\left(0^{e}, 0^{0}, 7^{d}, 9^{3}, d^{7}, c^{1}, c^{7}, d^{0}, 9^{b}, 6^{0}\right) & a^{0}:\left(9^{b}, d^{b}, 5^{9}, c^{b}, 6^{8}, 7^{2}, c^{9}, 5^{0}, d^{8}, 9^{0}\right) \\
3^{0}:\left(6^{c}, d^{8}, e^{0}, 5^{9}, 6^{9}, 0^{8}, 6^{6}, c^{9}, 6^{0}, 9^{b}\right) & b^{0}:\left(6^{0}, d^{b}, 9^{3}, 0^{3}, 5^{0}, 7^{2}, d^{0}, 9^{0}, 0^{0}, 5^{0}\right) \\
4^{0}:\left(7^{c}, c^{d}, 7^{6}, 0^{5}, 7^{3}, 5^{2}, e^{e}, d^{d}, 7^{0}, 9^{2}\right) & c^{0}:\left(1^{0}, 2^{8}, 8^{8}, 4^{2}, a^{6}, 1^{6}, 2^{e}, 8^{0}, 3^{6}, a^{4}\right) \\
5^{0}:\left(3^{6}, 4^{d}, b^{e}, 8^{b}, a^{6}, 1^{2}, 1^{0}, a^{0}, 8^{8}, a^{0}\right) & d^{0}:\left(a^{4}, b^{0}, 4^{2}, 3^{7}, b^{4}, a^{7}, 8^{0}, 2^{0}, 2^{8}, 8^{9}\right) \\
6^{0}:\left(3^{6}, b^{0}, 3^{3}, 1^{1}, 3^{9}, a^{7}, f^{3}, 8^{9}, 3^{0}, 2^{0}\right) & e^{0}:\left(3^{6}, 4^{2}, 3^{9}, 4^{5}, 3^{c}, 4^{8}, 3^{0}, 4^{b}, 3^{3}, 4^{e}\right) \\
7^{0}:\left(4^{9}, a^{d}, f^{3}, 8^{9}, 4^{3}, 2^{2}, 4^{c}, b^{d}, 4^{0}, 1^{0}\right) & f^{0}:\left(7^{0}, 6^{0}, 7^{3}, 6^{3}, 7^{6}, 6^{6}, 7^{9}, 6^{9}, 7^{c}, 6^{c}\right)
\end{array}
$$

Let $A=\left(A_{0}, A_{1}, \ldots, A_{g}\right), D=\left(D_{0}, D_{2}, \ldots, D_{f}\right), C=\left(C_{0}, C_{4}, \ldots, C_{d}\right), F=\left(F_{0}, F_{8}\right.$, $\left.\ldots, F_{9}\right)$ be 4 disjoint 17 -cycles. Each $y=A, D, C, F$ has vertices $y_{i}$ with $i$ expressed as an heptadecimal index up to $g=16$. We assume that $i$ is advancing in 1,2,4,8 units mod 17 , stepwise from left to right, respectively for $y=A, D, C, F$. Then the Biggs-Smith graph $B$-S is obtained by adding to the disjoint union $A \cup D \cup C \cup F$, for each $i \in \mathbf{Z}_{17}$, a 6 -vertex tree $T_{i}$ formed by the edge-disjoint union of paths $A_{i} B_{i} C_{i}, D_{i} E_{i} F_{i}$, and $B_{i} E_{i}$, where the vertices $A_{i}, D_{i}, C_{i}, F_{i}$ are already present in the cycles $A, D, C, F$, respectively, and where the vertices $B_{i}$ and $E_{i}$ are new and introduced with the purpose of defining the tree $T_{i}$, for $0 \leq i \leq g=16$. Now, $\mathcal{S}$ has the collection $\mathcal{C}_{9}$ of 9 -cycles formed by:

$$
\begin{array}{l|l}
S^{0}=\left(A_{0} A_{1} B_{1} C_{1} C_{5} C_{9} C_{d} C_{0} B_{0}\right), & W^{0}=\left(A_{0} A_{1} B_{1} E_{1} F_{1} F_{9} F_{0} E_{0} B_{0}\right), \\
T^{0}=\left(C_{0} C_{4} B_{4} A_{4} A_{3} A_{2} A_{1} A_{0} B_{0}\right), & X^{0}=\left(C_{0} C_{4} B_{4} E_{4} D_{4} D_{2} D_{0} E_{0} B_{0}\right), \\
U^{0}=\left(E_{0} F_{0} F_{9} F_{1} F_{a} F_{2} E_{2} D_{2} D_{0},\right) & Y^{0}=\left(E_{0} B_{0} A_{0} A_{1} A_{2} B_{2} E_{2} D_{2} D_{0}\right), \\
V^{0}=\left(E_{0} D_{0} D_{2} D_{4} D_{6} D_{8} E_{8} F_{8} F_{0}\right), & Z^{0}=\left(F_{0} F_{8} E_{8} B_{8} C_{8} C_{4} C_{0} B_{0} E_{0}\right),
\end{array}
$$


and those 9-cycles obtained from these, as $S^{x}, \ldots, Z^{x}$, by uniformly adding $x \in \mathbf{Z}_{17}$ mod 17 to all subindexes $i$ of vertices $y_{i}$, so that $\left|\mathcal{C}_{9}\right|=136$.

An auxiliary table presenting the form in which the 9-cycles above share the vertex sets of 3-arcs, either O-O or not, is shown below, in a fashion similar to those above for Pet, Hea, Pap and Fos, where minus signs are set but plus signs are tacit now:

$$
\begin{array}{l|l}
S^{0}:\left(-T_{1}^{e}, T_{7}^{1},-Z_{4}^{1}, S_{4}^{d}, S_{3}^{4},-Z_{3}^{9}, T_{0}^{d},-T_{6}^{0}, U_{8}^{0}\right), & W^{0}:\left(-U_{4}^{0}, W_{2}^{8}, W_{1}^{9},-U_{3}^{1}, X_{7}^{2},-X_{4}^{b}, Y_{6}^{0},-X_{8}^{0}, X_{5}^{9}\right), \\
T^{0}:\left(S_{6}^{4},-S_{0}^{3},-Y_{2}^{2}, T_{4}^{1}, T_{3}^{g},-Y_{1}^{0},-S_{7}^{0}, S_{1}^{g}, V_{8}^{0}\right), & X^{0}:\left(-V_{4}^{0}, X_{2}^{f}, X_{1}^{2},-V_{3}^{4},-W_{5}^{6}, W_{8}^{8},-Z_{8}^{0}, W_{4}^{f},-W_{7}^{0}\right), \\
U^{0}:\left(Y_{3}^{g},-U_{6}^{1}, Z_{7}^{1},-W_{3}^{g},-W_{0}^{0}, Z_{0}^{9},-U_{1}^{g}, Y_{0}^{0}, S_{8}^{0}\right), & Y^{0}:\left(U_{7}^{0},-T_{5}^{0},-T_{2}^{f}, U_{0}^{1},-Y_{8}^{2}, V_{2}^{f}, W_{6}^{0}, V_{5}^{0},-Y_{4}^{f}\right), \\
V^{0}:\left(-Z_{2}^{d},-V_{6}^{4}, Y_{5}^{2},-X_{3}^{d},-X_{0}^{0}, Y_{7}^{0},-V_{1}^{d},-Z_{5}^{0}, T_{8}^{0}\right), & Z^{0}:\left(U_{5}^{8},-Z_{6}^{8},-V_{0}^{4},-S_{5}^{8},-S_{2}^{g},-V_{7}^{0},-Z_{1}^{9}, U_{2}^{g},-X_{6}^{0}\right),
\end{array}
$$

This table is extended by adding $x \in \mathbf{Z}_{17}$ uniformly mod 17 to all superindexes, confirming that $B-S$ is not $\left\{\vec{C}_{9}\right\}_{\vec{P}_{4}}$-UH..

\section{Separator digraphs of 7 CDT graphs}

For each of the 7 CDT graphs $G$ that are $\left\{\overrightarrow{C_{g}}\right\}_{P_{k}}$-UH digraphs according to Theorem 3, the following construction yields a corresponding digraph $\mathcal{S}(G)$ of outdegree and indegree two and having underlying cubic graph structure and the same automorphism group of $G$. The vertices of $\mathcal{S}(G)$ are defined as the $(k-1)$-arcs of $G$. We set an arc in $\mathcal{S}(G)$ from each vertex $a_{1} a_{2} \ldots a_{k-1}$ into another vertex $a_{2} \ldots a_{k-1} a_{k}$ whenever there is an oriented $g$-cycle $\left(a_{1} a_{2} \ldots a_{k-1} a_{k} \ldots\right)$ in the $\left\{\eta \vec{C}_{g}\right\}_{\vec{P}_{k}}$-OOC provided by Theorem 3 to $G$. Thus each oriented $g$-cycle in the mentioned $\left\{\eta \vec{C}_{g}\right\}_{\vec{P}_{k}}$-OOC yields an oriented $g$-cycle of $\mathcal{S}(G)$. In addition we set an edge $e$ in $\mathcal{S}(G)$ for each transposition of a $(k-1)$-arc of $G$, say $h=a_{1} a_{2} \ldots a_{k-1}$, taking it into $h^{-1}=a_{k-1} a_{k-2} \ldots a_{1}$. Thus the ends of $e$ are $h$ and $h^{-1}$. As usual, the edge $e$ is considered composed by two O-O arcs.

The polyhedral graphs $G$ here are the tetrahedral graph $G=K_{4}$, the 3-cube graph $G=Q_{3}$ and the dodecahedral graph $G=\Delta$. The corresponding graphs $\mathcal{S}(G)$ have their underlying graphs respectively being the truncated-polyhedral graphs of the corresponding dualpolyhedral graphs that we can refer as the truncated tetrahedron, the truncated octahedron and the truncated icosahedron. In fact:

(A) $\mathcal{S}\left(K_{4}\right)$ has vertices $01,02,03,12,13,23,10,20,30,21,31,32$; the cycles $(123),(210)$, (301), (032) of the $\left\{\eta \vec{C}_{g}\right\}_{\vec{P}_{k}}$-OOC of $K_{4}$ give place to the oriented 3-cycles $(12,23$, $31),(21,10,02),(30,01,13),(03,32,20)$ of $\mathcal{S}\left(K_{4}\right)$; the additional edges of $\mathcal{S}\left(K_{4}\right)$ are $(01,10),(02,20),(03,30),(12,21),(13,31),(23,32)$.

(B) The of oriented cycles of $\mathcal{S}\left(Q_{3}\right)$ corresponding to the $\left\{\eta \vec{C}_{g}\right\}_{\vec{P}_{k}}$-OOC of $Q_{3}$ are $(01,13$, $32,20),(10,04,45,51),(31,15,57,73),(23,37,76,62),(02,26,64,40),(46,67,75,54)$; the additional edges of $\mathcal{S}\left(Q_{3}\right)$ are $(01,10),(23,32),(45,54),(67,76),(02,20),(13,31)$, $(46,64),(57,75),(04,40),(15,51),(26,62),(37,73)$.

(C) The oriented cycles of $\mathcal{S}(\Delta)$ corresponding to the $\left\{\eta \vec{C}_{g}\right\}_{\vec{P}_{k}}$-OOC of $\Delta$ are $\left(a_{0} a_{1}, a_{1} a_{2}\right.$, $\left.a_{2} a_{3}, a_{3} a_{4}, a_{4} a_{0}\right),\left(c_{4} c_{3}, c_{3} c_{2}, c_{2} c_{1}, c_{1} c_{0}, c_{0} c_{4}\right)$, and both $\left(a_{x} d_{x}, d_{x} b_{x-2}, b_{x-2} d_{x+1}, d_{x+1}\right.$ $\left.a_{x+1}, a_{x+1} a_{x}\right)$ and $\left(d_{x} b_{x+2}, b_{x+2} c_{x+2}, c_{x+2} c_{x-2}, c_{x-2} b_{x-2}, b_{x-2} d_{x}\right)$, for each $x \in \mathbf{Z}_{5}$; 

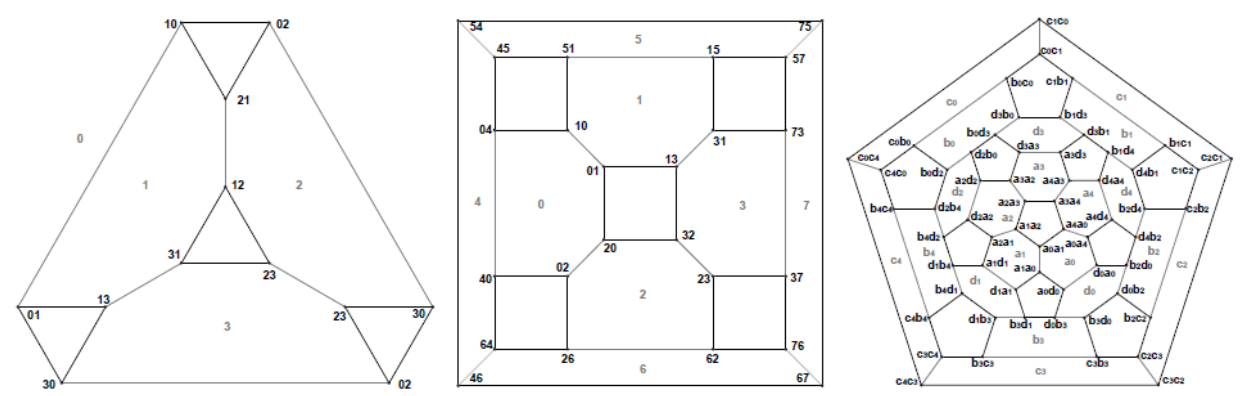

Figure 1: $\mathcal{S}\left(K_{4}\right), \mathcal{S}\left(Q_{3}\right)$ and $\mathcal{S}(\Delta)$

the additional edges are $\left(a_{x} a_{x+1}, a_{x+1} a_{x}\right),\left(c_{x} c_{x+1}, c_{x+1} c_{x}\right),\left(a_{x} d_{x}, d_{x} a_{x}\right),\left(d_{x} b_{x+2}\right.$, $\left.b_{x+2} d_{x}\right),\left(b_{x} d_{x+2}, d_{x+2} b_{x}\right)$, and $\left(b_{x} c_{x}, c_{x} b_{x}\right)$, for each $x \in \mathbf{Z}_{5}$.

Among the 7 CDT graphs $G$ that are $\left\{\overrightarrow{C_{g}}\right\}_{P_{k}}$-UH digraphs, the polyhedral graphs treated above are exactly those having arc-transitivity $k=2$. Figure 1 contains representations of these graphs $\mathcal{S}(G)$, namely $\mathcal{S}\left(K_{4}\right), \mathcal{S}\left(Q_{3}\right)$ and $\mathcal{S}(\Delta)$, with the respective 3-cycles, 4-cycles and 5-cycles in black trace to be considered clockwise oriented, but for the external cycles in the cases $\mathcal{S}\left(Q_{3}\right)$ and $\mathcal{S}(\Delta)$, to be considered counterclockwise oriented. The remaining edges (to be referred as transposition edges) are gray colored and considered bidirectional. The cycles having alternate black and gray edges here, arising respectively from arcs from the oriented cycles and from the transposition edges, are 6-cycles. Each such 6-cycle has its vertices sharing the notation, indicated in gray, of a unique vertex of the corresponding $G$. Each vertex of $G$ is used as such gray 6-cycle indication.

The truncated tetrahedron, truncated octahedron and truncated icosahedron, oriented as indicated for Figure 1, are the Cayley digraphs of the groups $A_{4}, S_{4}$ and $A_{5}$, with respective generating sets $\{(123),(12)(34)\},\{(1234),(12)\}$ and $\{(12345),(23)(45)\}$. Thus

$$
\mathcal{S}\left(K_{4}\right) \equiv \operatorname{Cay}\left(A_{4},\{(123),(12)(34)\}\right), \mathcal{S}\left(Q_{3}\right) \equiv \operatorname{Cay}\left(S_{4},\{(1234),(12)\}\right), \mathcal{S}(\Delta) \equiv \operatorname{Cay}\left(A_{5},\{(12345),(23)(45)\}\right) .
$$

(D) The oriented cycles of $\mathcal{S}\left(K_{3,3}\right)$ corresponding to the $\left\{\eta \vec{C}_{g}\right\}_{\vec{P}_{k}}$-OOC of $K_{3,3}$ are:

$$
\begin{array}{lll}
(123,234,341,412), & (321,210,103,032), & (432,325,254,543), \\
(143,430,301,014), & (214,145,452,521), & (012,125,250,501), \\
(523,230,305,052), & (034,345,450,503), & (541,410,105,054) ;
\end{array}
$$

and additional edges of $\mathcal{S}\left(K_{3,3}\right)$ are:

$(123,321),(234,432),(341,143),(412,214),(210,012),(103,301)$,

$(032,230),(325,523),(254,452),(543,345),(430,034),(014,410)$,

$(145,541),(521,125),(250,052),(501,105),(305,503),(450,054)$. 


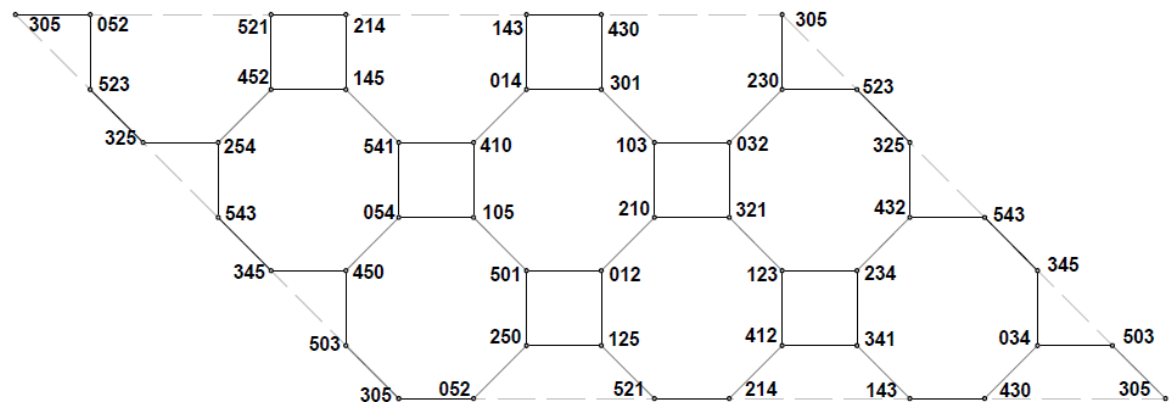

Figure 2: $\mathcal{S}\left(K_{3,3}\right)$

A cut-out toroidal representation of $\mathcal{S}\left(K_{3,3}\right)$ is in Figure 2, where black-traced 4-cycles are considered oriented clockwise, corresponding to the oriented 4-cycles in the $\left\{\eta \vec{C}_{g}\right\}_{\vec{P}_{k}}{ }^{-}$ OOC of $K_{3,3}$, and where gray edges represent transposition edges of $\mathcal{S}\left(K_{3,3}\right)$, which gives place to the alternate 8 -cycles, constituted each by an alternation of 4 transposition edges and 4 arcs of the oriented 4-cycles. In $\mathcal{S}\left(K_{3,3}\right)$, there are 94 -cycles and 98 -cycles. Now, the group $<(0,5,4,1)(2,3),(0,2)(1,5)>$ has order 36 and acts regularly on the vertices of $\mathcal{S}\left(K_{3,3}\right)$. For example, $(0,2)(1,5)$ stabilizes the edge $(145,541)$, and the permutation $(0,5,4,1)(2,3)$ permutes (clockwise) the black oriented 4-cycle $(541,410,105,054)$. Thus $\mathcal{S}\left(K_{3,3}\right)$ is a Cayley digraph. Also, observe the oriented 9-cycles in $\mathcal{S}\left(K_{3,3}\right)$ obtained by traversing alternatively 2 -arcs in the oriented 4 -cycles and transposition edges; there are 6 such oriented 9-cycles.

(E) The collection of oriented cycles of $\mathcal{S}(D e s)$ corresponding to the $\left\{\eta \vec{C}_{g}\right\}_{\vec{P}_{k}}$-OOC of Des is formed by the following oriented 6-cycles, where $x \in \mathbf{Z}_{5}$ :

$$
\begin{aligned}
& \left(x_{0} x_{1} x_{2}, x_{1} x_{2} x_{3}, x_{2} x_{3} x_{0}^{1}, x_{3} x_{0}^{1} x_{3}^{4}, x_{0}^{1} x_{3}^{4} x_{0}, x_{3}^{4} x_{0} x_{1}\right), \\
& \left(x_{1} x_{0} x_{3}^{4}, x_{0} x_{3}^{4} x_{2}^{4}, x_{3}^{4} x_{2}^{4} x_{1}^{2}, x_{2}^{4} x_{1}^{2} x_{2}^{2}, x_{1}^{2} x_{2}^{2} x_{1}, x_{2}^{2} x_{1} x_{0}\right), \\
& \left(x_{2} x_{1} x_{0}, x_{1} x_{0} x_{3}^{3}, x_{0} x_{3}^{3} x_{2}^{3}, x_{3}^{3} x_{2}^{3} x_{1}^{3}, x_{2}^{3} x_{1}^{3} x_{2}, x_{1}^{3} x_{2} x_{1}\right), \\
& \left(x_{0} x_{3}^{4} x_{0}^{1}, x_{3}^{4} x_{0}^{1} x_{1}^{1}, x_{0}^{1} x_{1}^{1} x_{2}^{3}, x_{1}^{1} x_{2}^{3} x_{3}^{3}, x_{2}^{3} x_{3}^{3} x_{0}, x_{3}^{3} x_{0} x_{3}^{4}\right),
\end{aligned}
$$

respectively for the 6-cycles $A^{x}, B^{x}, C^{x}, D^{x}$, where $x_{i}^{j}$ stands for $(x+j)_{i}$; each of the participant vertices here is an end of a transposition edge. Figure 3 represents a subgraph $\mathcal{S}\left(M_{3}\right)$ of $\mathcal{S}($ Des $)$ associated with the matching $M_{3}$ of Des indicated in its representation "inside" the left-upper "eye" of the figure, where vigesimal integer notation is used (up to $j=19$ ); in the figure, additional intermittent edges were added that form 12 square pyramids, 4 such edges departing from a corresponding extra vertex; so, 12 extra vertices appear that can be seen as the vertices of a cuboctahedron whose edges are 3-paths with inner edge in $\mathcal{S}\left(M_{3}\right)$ and intermittent outer edges. There is a total of 5 matchings, like $M_{3}$, that we denote $M_{x}$, where $x=3,7, b, f, j$. In fact, $\mathcal{S}(D e s)$ is obtained as the union $\bigcup\left\{\mathcal{S}\left(M_{x}\right) ; x=3,7, b, f, j\right\}$. Observe that the components of the subgraph induced by the matching $M_{x}$ in Des are at mutual distance 2 and that $M_{x}$ can be divided into three pairs of edges with the ends of each pair at minimum distance 4 , facts that can be used to establish the properties of $\mathcal{S}(D e s)$. 


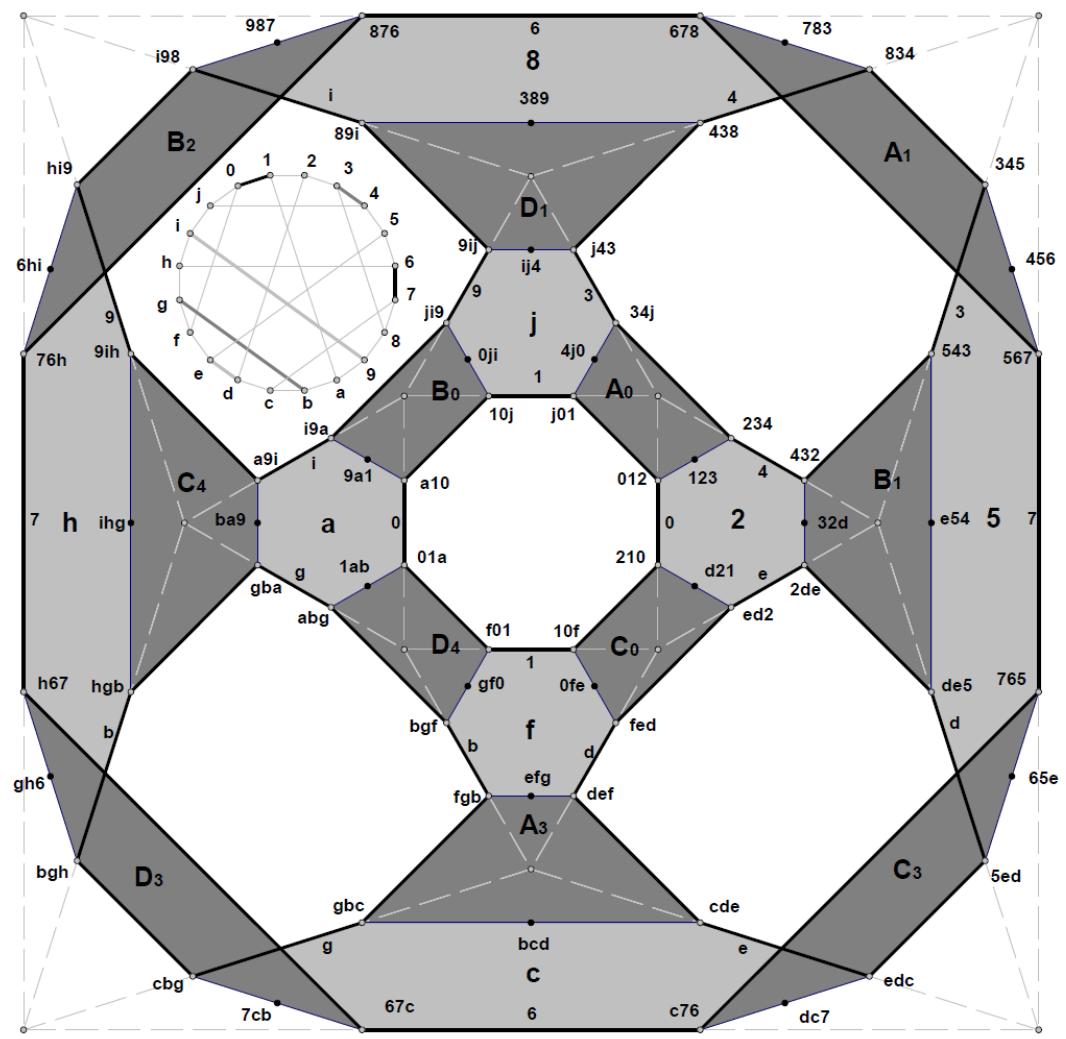

Figure 3: $M_{3}$ and the subgraph of $\mathcal{S}(D e s)$ associated to it 
In Figure 3 there are: 12 oriented 6-cycles (dark-gray interiors); 6 alternate 8-cycles (thickblack edges); and 89 -cycles with alternate 2 -arcs and transposition edges (light-gray interiors). The 6-cycles are denoted by means of the associated oriented 6-cycles of Des. Each 9-cycle has its vertices sharing the notation of a vertex of $V(D e s)$ and this is used to denote it. Each edge $e$ in $M_{3}$ has associated a closed walk in Des containing every 3-path with central edge $e$; this walk can be used to determine a unique alternate 8-cycle in $\mathcal{S}\left(M_{3}\right)$, and viceversa. Each 6-cycle has two opposite (black) vertices of degree two in $\mathcal{S}\left(M_{3}\right)$. In all, $\mathcal{S}($ Des $)$ contains 120 vertices; 360 arcs amounting to 120 arcs in oriented 6-cycles and 120 transposition edges; 20 dark-gray 6-cycles; 30 alternate 8-cycles; and 20 light-gray 9-cycles. By filling the 6-cycles and 8-cycles here with 2-dimensional faces, then the 120 vertices, 180 edges (of the underlying cubic graph) and resulting $20+30=50$ faces yield a surface of Euler characteristic $120-180+50=-10$, so this surface genus is 6 . The automorphism group of Des is $G=S_{5} \times \mathbf{Z}_{2}$. Now, $G$ contains three subgroups of index 2: two isomorphic to to $S_{5}$ and one isomorphic to $A_{5} \times \mathbf{Z}_{2}$. One of the two subgroups of $G$ isomorphic to $S_{5}$ (the diagonal copy) acts regularly on the vertices of $\mathcal{S}(D e s)$ and hence $\mathcal{S}(D e s)$ is a Cayley digraph.

(F) The collection of oriented cycles of $\mathcal{S}(C o x)$ corresponding to the $\left\{\eta \vec{C}_{g}\right\}_{\vec{P}_{k}}$-OOC of Cox is formed by oriented 7-cycles, such as:

$$
\underline{0^{1}}=\left(u_{1} u_{2} u_{3}, u_{2} u_{3} u_{4}, u_{3} u_{4} u_{5}, u_{4} u_{5} u_{6}, u_{5} u_{6} u_{0}, u_{6} u_{0} u_{1}, u_{0} u_{1} u_{2}\right)
$$

and so on for the remaining oriented 7-cycles $\underline{x^{y}}$ with $x \in\{0, \ldots, 7\}$ and $y \in\{1,2,3\}$, based on the corresponding table in the proof of Theorem 3. Moreover, each vertex of $\mathcal{S}(C o x)$ is adjacent via a transposition edge to its reversal vertex. Thus $\mathcal{S}(C o x)$ has: underlying cubic graph; indegree $=$ outdegree $=2 ; 168$ vertices; 168 arcs in 24 oriented 7-cycles; 84 transposition edges; and 42 alternate 8 -cycles. Its underlying cubic graph has 252 edges. From this information, by filling the 7- and 8-cycles mentioned above with 2-dimensional faces, we obtain a surface with Euler characteristic $168-252+(24+42)=-18$, so its genus is 10. On the other hand, $\mathcal{S}(C o x)$ is the Cayley digraph of the automorphism group of the Fano plane, namely $P S L(2,7)=G L(3,2)$ [4], of order 168, with a generating set of two elements, of order 2 and 7 , representable by the $3 \times 3$-matrices $(100,001,010)^{T}$ and $(001,101,010)^{T}$ over the field $F_{2}$, where $T$ stands for transpose.

Figure 4 depicts a subgraph of $\mathcal{S}(C o x)$ containing in its center a (twisted) alternate 8-cycle that we denote (in gray) $z_{1} u_{1}$, and, around it, four oriented 7-cycles adjacent to it, (namely $\underline{1^{1}}, \underline{7^{1}}, \underline{2^{3}}, \underline{6^{3}}$, denoted by their corresponding oriented 7-cycles in Cox, also in gray), plus four additional oriented 7-cycles (namely $\underline{0^{0}}, \underline{3^{2}}, \underline{4^{1}}, \underline{5^{2}}$ ), related to four 9-cycles mentioned below. Black edges represent arcs, and the orientation of these 87 -cycles is taken clockwise, with only gray edges representing transposition edges of $\mathcal{S}(C o x)$. Each edge of Cox determines an alternate 8-cycle of $\mathcal{S}(C o x)$. In fact, Figure 4 contains not only the alternate 8 -cycle corresponding to the edge $z_{1} u_{1}$ mentioned above, but also those corresponding to the edges $u_{1} u_{2}, v_{1} z_{1}, t_{1} z_{1}$ and $u_{0} u_{1}$. These 8-cycles and the 7-cycles in the figure show that alternate 8-cycles $C$ and $C^{\prime}$ adjacent to a particular alternate 8-cycle $C^{\prime \prime}$ in $\mathcal{S}(C o x)$ on opposite edges $e$ and $e^{\prime}$ of $C^{\prime \prime}$ have the same opposite edge $e^{\prime \prime}$ both to $e$ and $e^{\prime}$ in $C$ and $C^{\prime}$, respectively. There are two instances of this property in Figure 4, where the two edges taking the place of $e^{\prime \prime}$ are the large central diagonal gray ones, with $C^{\prime \prime}$ corresponding to $u_{1} z_{1}$. As in (E) above, the fact that each edge $e$ of Cox determines 


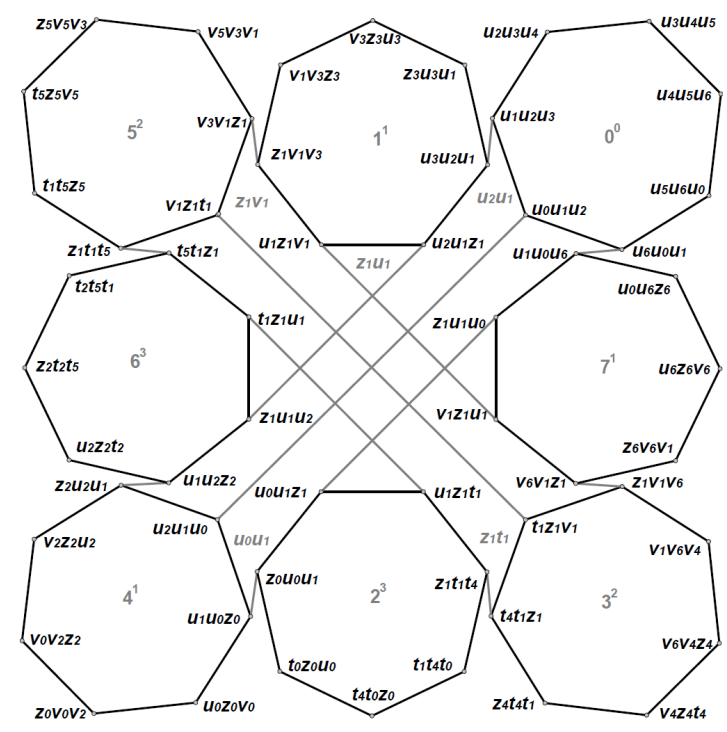

Figure 4: A subdigraph of $\mathcal{S}(C o x)$ associated with an edge of $C o x$

an alternate 8-cycle of $\mathcal{S}(C o x)$ is related with the closed walk that covers all the 3-paths having $e$ as central edge, and the digraph $\mathcal{S}(C o x)$ contains 9-cycles that alternate 2-arcs in the oriented 7-cycles with transposition edges. In the case of Figure 4, these 9-cycles are, in terms of the orientation of the 7-cycles:

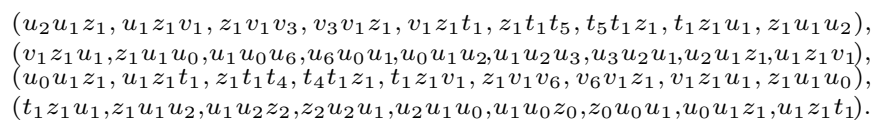

A convenient description of alternate 8-cycles, as those denoted in gray in Figure 4 by the edges $z_{1} u_{1}, z_{1} v_{1}, u_{2} u_{1}, u_{0} u_{1}, z_{1} t_{1}$ of $C o x$, is given by indicating the successive passages through arcs of the oriented 7-cycles, with indications by means of successive subindexes in the order of presentation of their composing vertices, which for those 5 alternate 8-cycles looks like:

$$
\left(1_{60}^{1}, 7_{56}^{1}, 2_{60}^{3}, 6_{56}^{3}\right), \quad\left(5_{12}^{2}, 3_{23}^{2}, 7_{45}^{1}, 1_{01}^{1}\right), \quad\left(0_{01}^{1}, 1_{56}^{1}, 6_{60}^{3}, 4_{56}^{1}\right), \quad\left(2_{56}^{3}, 7_{60}^{1}, 0_{56}^{0}, 4_{60}^{1}\right), \quad\left(3_{12}^{2}, 5_{23}^{2}, 6_{45}^{3}, 2_{01}^{3}\right)
$$

In a similar fashion, the four bi-alternate 9-cycles displayed just above can be presented by means of the shorter expressions:

$$
\left(1_{61}^{1}, 5_{13}^{2}, 6_{46}^{3}\right), \quad\left(7_{50}^{1}, 0_{50}^{1}, 1_{50}^{1}\right), \quad\left(2_{61}^{3}, 3_{13}^{2}, 7_{46}^{1}\right), \quad\left(6_{50}^{3}, 4_{50}^{1}, 2_{50}^{3}\right) .
$$

By the same token, there are twenty four tri-alternate 28-cycles, one of which is expressible as:

$$
\left(0_{03}^{1}, 6_{03}^{1}, 3_{40}^{2}, 2_{36}^{3}, 5_{36}^{3}, 4_{62}^{2}, 1_{40}^{1}\right)
$$


At this point, we observe that $\mathcal{S}\left(K_{4}\right), \mathcal{S}\left(Q_{3}\right)$ and $\mathcal{S}(\Delta)$ have alternate 6-cycles, while $\mathcal{S}\left(K_{3,3}\right), \mathcal{S}(D e s)$ and $\mathcal{S}($ Cox $)$ have alternate 8-cycles.

(G) The collection of oriented cycles of $\mathcal{S}(T u t)$ corresponding to the $\left\{\eta \vec{C}_{g}\right\}_{\vec{P}_{k}}$-OOC of Tut is formed by oriented 8-cycles, such as: $\underline{A^{0}}=$

$\left(4_{5} 0_{0} 0_{1} 0_{2} 0_{3}, 0_{0} 0_{1} 0_{2} 0_{3} 0_{4}, 0_{1} 0_{2} 0_{3} 0_{4} 0_{5}, 0_{2} 0_{3} 0_{4} 0_{5} 1_{0}, 0_{3} 0_{4} 0_{5} 1_{0} 4_{5}, 0_{4} 0_{5} 1_{0} 4_{5} 0_{0}, 0_{5} 1_{0} 4_{5} 0_{0} 0_{1}, 1_{0} 4_{5} 0_{0} 0_{1} 0_{2}\right)$

and so on for the remaining oriented 8-cycles $\underline{X^{y}}$ with $X \in\{A, \ldots, R\}$ and $y \in \mathbf{Z}_{5}$ based on the corresponding table in the proof of Theorem 3. Moreover, each vertex of $\mathcal{S}(T u t)$ is adjacent via a transposition edge to its reversal vertex. Thus $\mathcal{S}(T u t)$ has: underlying cubic graph; indegree $=$ outdegree $=2 ; 720$ vertices; 720 arcs in 90 oriented 8-cycles; 360 transposition edges; and 180 alternate 8-cycles, (36 of which are displayed below); its underlying cubic graph has 1080 edges. From this information, by filling the 9008 -cycles above with 2-dimensional faces, a surface with Euler characteristic $720-1080+240=$ -120 is obtained, so genus $=61$. On the other hand, the automorphism group of Tut is the projective semilinear group $G=P \Gamma L(2,9)$ [13], namely the group of collineations of the projective line $P G(1,9)$. The group $G$ contains exactly three subgroups of index 2 (and so of order 720), one of which (namely $M_{10}$, the Mathieu group of order 10, acts regularly on the vertices of $\mathcal{S}(T u t)$. Thus $\mathcal{S}(T u t)$ is a Cayley digraph.

A fifth of the 180 alternate 8-cycles of $\mathcal{S}(T u t)$ can be described by presenting in each case the successive pairs of vertices in each oriented 8-cycle $\underline{X^{y}}$ as follows, each such pair denoted by means of the notation $X_{u(u+1)}^{y}$, where $u$ stands for the 4-arc in position $u$ in $\underline{X^{y}}$, with 0 indicating the first position:

\begin{tabular}{|c|c|c|c|c|c|c|c|c|c|c|c|}
\hline$\left(A_{01}^{0}\right.$ & $M_{34}^{0}$, & $B_{34}^{4}$, & $\left.K_{12}^{0}\right)$ & $\left(A_{12}^{0}\right.$, & $P_{01}^{1}$, & $I_{70}^{0}$ & $\left.M_{23}^{0}\right)$ & $\left(A_{23}^{0}\right.$, & $H_{34}^{0}$, & $B_{70}^{1}$, & $\left.P_{70}^{1}\right)$ \\
\hline & $J_{70}^{0}$ & $L_{70}^{3}$, & $\left.H_{23}^{0}\right)$ & $\left(A_{45}^{0}\right.$, & $E_{70}^{0}$, & $P_{45}^{0}$, & $\left.J_{67}^{0}\right)$ & $\left(A_{56}^{0}\right.$ & $E_{45}^{1}$, & $F_{70}^{1}$, & $\left.E_{67}^{0}\right)$ \\
\hline${ }^{6} 6$ & $G_{45}^{0}$, & $M_{67}^{1}$, & $\left.E_{34}^{(}\right)$ & $\left(A_{70}^{0^{\circ}}\right.$, & $K_{23}^{0}$, & $L_{12}^{2^{3}}$, & $\left.G_{34}^{0}\right)$ & $\left(B_{01}^{8^{\circ}}\right.$ & $C_{34}^{t^{2}}$, & $Q_{23}^{3}$, & $\left.P_{67}^{8}\right)$ \\
\hline 62 & $R_{34}^{3}$ & $N_{56}^{1}$, & $\left.C_{23}^{2}\right)$ & $\left(B_{23}^{0}\right.$, & $M_{45}^{1}$ & $Q_{67}^{2}$ & $\left.R_{23}^{3}\right)$ & $\left(B_{45}^{0}\right.$ & $D_{67}^{3}$, & $R_{70}^{1}$ & $\left.K_{01}^{1}\right)$ \\
\hline $3_{5}^{0}$ & $D_{34}^{0}$, & $O_{56}^{0}$, & $\left.D_{56}^{3}\right)$ & $\left(B_{67}^{0}\right.$ & $H_{45}^{4}$, & $C_{67}^{4}$, & $\left.D_{23}^{\gamma^{\circ}}\right)$ & $\left(C_{0}^{0}\right.$ & $G_{70}^{3}$, & $H_{70}^{3}$ & $\left.M_{01}^{0}\right)$ \\
\hline 12 & $N_{67}^{4}$, & $F_{45}^{3}$, & $\left.G_{67}^{3}\right)$ & $\left(C_{45}^{0}\right.$, & $L_{67}^{0}$ & $J_{01}^{2}$ & $\left.Q_{12}^{1}\right)$ & $\left(C_{56}^{0}\right.$, & $H_{56}^{0}$, & $O_{34}^{4}$, & $\left.L_{56}^{0}\right)$ \\
\hline$\left(C_{70}^{0}\right.$, & $M_{12}^{0}$, & $I_{01}^{0}$ & $\left.D_{12}^{1}\right)$ & $\left(D_{01}^{0}\right.$, & $I_{12}^{4}$ & $O_{12}^{1}$ & $\left.I_{56}^{3}\right)$ & $\left(D_{45}^{0}\right.$, & $O_{67}^{2}$ & $L_{45}^{\mathrm{P}^{\mathrm{T}}}$, & $\left.O_{45}^{0}\right)$ \\
\hline$\left(D_{\gamma^{0}}^{0}\right.$, & $I_{67}^{3}$ & $P_{12}^{4}$, & $\left.R_{6,7}^{3}\right)$ & $\left(E_{01}^{0}\right.$, & $N_{01}^{4}$, & $K_{45}^{1}$ & $\begin{array}{l}\left.P_{34}^{0}\right) \\
F^{0}\end{array}$ & $\left(E_{12}^{0}\right.$, & $N_{34}^{2}$ & $F_{34}^{3}$ & $\left.N_{70}^{4}\right)$ \\
\hline $\begin{array}{l}\left(E_{23}^{0},\right. \\
F^{0},\end{array}$ & $\begin{array}{l}M_{70}^{\mathrm{O}} \\
N^{4}\end{array}$ & $\begin{array}{l}H_{01}^{3}, \\
R^{1},\end{array}$ & $\begin{array}{c}\left.N_{233}^{2}\right) \\
\left.J^{3}\right)\end{array}$ & $\begin{array}{l}\left(E_{56}^{\mathrm{o}},\right. \\
\left(F^{0},\right.\end{array}$ & $F_{01}^{1}$, & $Q_{4}^{2}$ & $\begin{array}{l}\left.F_{67}^{0}\right) \\
G^{0}\end{array}$ & & $J_{56}^{3}$ & $\begin{array}{l}P_{56}^{3}, \\
O^{4}\end{array}$ & $\begin{array}{l}\left.Q_{34}^{1}\right) \\
H^{0}\end{array}$ \\
\hline$G_{12}$ & $\begin{array}{l}N_{45}, \\
Q_{01}^{45},\end{array}$ & $\begin{array}{l}T_{45}^{2}, \\
J_{12}^{2}\end{array}$ & $\begin{array}{l}\left.J_{45}\right) \\
\left.I_{34}^{1}\right)\end{array}$ & $\left(G_{23}^{0}\right.$, & $\begin{array}{l}Q_{56}^{2} \\
L_{23}^{2},\end{array}$ & $\begin{array}{l}M_{5}{ }^{2} \\
R_{12}^{2}\end{array}$ & $\begin{array}{l}\left.G_{56}\right) \\
\left.Q_{70}^{1}\right)\end{array}$ & $\left(H_{12}^{0}\right.$, & $\begin{array}{l}I_{45}, \\
L_{01}^{3},\end{array}$ & $\begin{array}{l}23 \\
K_{34}^{13},\end{array}$ & $\begin{array}{l}\left.H_{67}\right) \\
\left.N_{12}^{4}\right)\end{array}$ \\
\hline 0 & $J_{23}^{1}$ & $K_{67}^{1}$, & $\left.O_{01}^{2}\right)$ & $\left(J_{34}^{0}\right.$ & $R_{56}^{3}$ & $P_{23}^{4}$ & $\left.K_{56}^{0}\right)$ & $\left(K_{70}^{0}\right.$ & $R_{01}^{0}$ & $L_{34}^{0}$, & $\left.O_{70}^{1}\right)$ \\
\hline
\end{tabular}

Again, as in the previously treated cases, we may consider the oriented paths that alternatively traverse two arcs in an oriented 8-cycle and then a transposition edge, repeating this operation until a closed path is formed. It happens that all such bi-alternate cycles are 12-cycles. For example with a notation akin to the one in the last table, we display the first row of the corresponding table of 12-cycles:

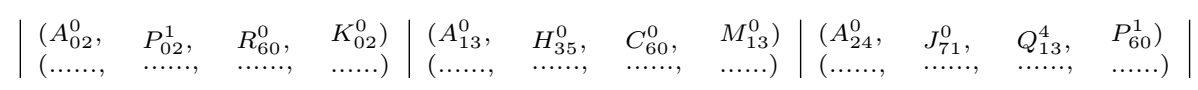

As in the case of the alternate 8-cycles above, which are 180, there are 180 bi-alternate 12 -cycles in $\mathcal{S}($ Tut $)$. On the other hand, an example of a tri-alternate 32-cycle in $\mathcal{S}($ Tut $)$ is given by:

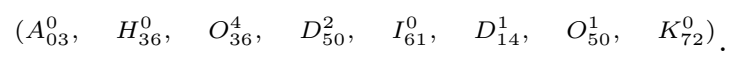


There is a total of 90 such 32-cycles. Finally, an example of a tetra-alternate 15-cycle in $\mathcal{S}(T u t)$ is given by $\left(A_{04}^{0}, J_{73}^{0}, K_{62}^{0}\right)$, and there is a total of 240 such 15 -cycles. More can be said about the relative structure of all these types of cycles in $\mathcal{S}(T u t)$.

The automorphism groups of the graphs $\mathcal{S}(G)$ in items (A)-(G) above coincide with those of the corresponding graphs $G$ because the construction of $\mathcal{S}(G)$ depends solely on the structure of $G$ as analyzed in Section 3 above. Salient properties of the graphs $\mathcal{S}(G)$ are contained in the following statement.

Theorem 4.1. For each CDT graph that is a $\left\{\overrightarrow{C_{g}}\right\}_{P_{k}}$-UH digraph, $\mathcal{S}(G)$ is: (a) a vertextransitive digraph with indegree $=$ outdegree $=2$, underlying cubic graph and the automorphism group of $G$; (b) a $\left\{\vec{C}_{g}, \vec{C}_{2}\right\}$-ultrahomogeneous digraph, where $\vec{C}_{g}$ stands for oriented $g$-cycle coincident with its induced subdigraph and each vertex is the intersection of exactly one such $\overrightarrow{C_{g}}$ and one $\overrightarrow{C_{2}}$; (c) a Cayley digraph. Moreover, the following additional properties hold, where $s(G)=$ subjacent undirected graph of $\mathcal{S}(G)$ :

(A) $\mathcal{S}\left(K_{4}\right) \equiv \operatorname{Cay}\left(A_{4},\{(123),(12)(34)\}\right), s\left(K_{4}\right)=$ truncated octahedron;

(B) $\mathcal{S}\left(Q_{3}\right) \equiv \operatorname{Cay}\left(S_{4},\{(1234),(12)\}\right), s\left(Q_{3}\right)=$ truncated octahedron;

(C) $\mathcal{S}(\Delta) \equiv \operatorname{Cay}\left(A_{5},\{(12345),(23)(45)\}\right), s(\Delta)=$ truncated icosahedron;

(D) $\mathcal{S}\left(K_{3,3}\right)$ is the Cayley digraph of the subgroup of $S_{6}$ on the vertex set $\{0,1,2,3,4,5\}$ generated by $(0,5,4,1)(2,3)$ and $(0,2)(1,5)$ and has a toroidal embedding whose faces are delimited by 9 oriented 4 -cycles and 9 alternate 8 -cycles;

(E) $\mathcal{S}($ Des $)$ is the Cayley digraph of a diagonal copy of $S_{5}$ in the automorphism group $S_{5} \times \mathbf{Z}_{2}$ of Des and has a 6 -toroidal embedding whose faces are delimited by 20 oriented 6 -cycles and 30 alternate 8 -cycles;

(F) $\mathcal{S}(\operatorname{Cox}) \equiv \operatorname{Cay}\left(G L(3,2),\left\{(100,001,010)^{T},(001,101,010)^{T}\right\}\right)$, has a 10-toroidal embedding whose faces are delimited by 24 oriented 7 -cycles and 42 alternate 8 cycles;

(G) $\mathcal{S}($ Tut $)$ is the Cayley digraph of a subgroup $M_{10}$ of order 2 in the automorphism group $P \Gamma L(2,9)$ of $T u t$ and has a 61 -toroidal embedding whose faces are delimited by 90 oriented 8 -cycles and 180 alternate 8 -cycles.

Corollary 4.2. The bi-alternate cycles in the graphs $\mathcal{S}(G)$ above are 9 -cycles unless either $G=Q_{3}$ or $G=\Delta$, in which cases they are respectively 12 -cycles and 15 -cycles.

\section{Acknowledgement}

The author is grateful to the referee of a previous version of this paper for indications and corrections that lead to the present manuscript and for encouragement with respect to the value of the construction of the 7 directed graphs $\mathcal{S}(G)$ in Section 4 .

\section{References}

[1] N. L. Biggs, Algebraic Graph Theory (2nd ed.), Cambridge University Press, 1993.

[2] B. Mohar and C. Thomassen, Graphs on surfaces, Johns Hopkins Univ. Press, 2001. 
[3] A. E. Brouwer, A. M. Cohen and A. Neumaier, Distance-Regular Graphs, Springer-Verlag, New York 1989.

[4] E. Brown and N. Loehr, Why is $\operatorname{PSL}(2,7)=G L(3,2)$ ?, Amer. Math. Mo. 116-8 (2009), 727-732.

[5] P. J. Cameron, 6-transitive graphs, J. Combin. Theory Ser. B 28 (1980), 168-179.

[6] G. L. Cherlin, The Classification of Countable Homogeneous Directed Graphs and Countable Homogeneous $n$-tournaments, Memoirs Amer. Math. Soc., vol. 131, number 612, Providence RI, January 1988.

[7] A. Devillers, Classification of some homogeneous and ultrahomogeneous structures, Dr. Sc. Thesis, Université Libre de Bruxelles, 2001-2002.

[8] R. Fraïssé, Sur l'extension aux relations de quelques proprietés des ordres, Ann. Sci. École Norm. Sup. 71 (1954), 363-388.

[9] A. Gardiner, Homogeneous graphs, J. Combin. Theory Ser. B 20 (1976), 94-102.

[10] C. Godsil and G. Royle, Algebraic Graph Theory, Springer-Verlag, 2001.

[11] Ja. Ju. Gol'fand and M. H. Klin, On $k$-homogeneous graphs, Algorithmic studies in combinatorics 186 (1978), 76-85, in Russian.

[12] J. L. Gross and T. W. Tucker, Topological Graph Theory, Wiley Interscience, 1987.

[13] J. W. P. Hirschfeld, Projective Geometries over Finite Fields, Oxford Science Publications, 2nd ed., Clarendom Press, Oxford 1998.

[14] D. C. Isaksen, C. Jankowski and S. Proctor, On $K_{*}$-ultrahomogeneous graphs, Ars Combinatoria 82 (2007), 83-96.

[15] I. A. Faradzev, A. A. Ivanov, M. Klin et al., The Algebraic Theory of Combinatorial Objects, Math. Appl. (Soviet Series), 84, Dordrecht, Kluwer, 1992.

[16] A. H. Lachlan and R. Woodrow, Countable ultrahomogeneous undirected graphs, Trans. Amer. Math. Soc. 262 (1980), 51-94.

[17] C. Ronse, On homogeneous graphs, J. London Math. Soc. 17 (1978), 375-379.

[18] B. I. Rose and R. E. Woodrow, Ultrahomogeneous structures, Mathematical Logic Quarterly 27 (1981), 23-30.

[19] J. Sheehan, Smoothly embeddable subgraphs, J. London Math. Soc. 9 (1974), 212-218.

[20] A. T. White, Graphs of groups on surfaces, North-Holland, Mathematics Studies 188, 2001. 